Araneda, O.F.; Calderón A.; Terreros, L. y Cavada, G. (2020) Swim 2,5-Km Indoor Decreases the Nitrite and $\mathrm{Ph}$ in Exhaled Breath Condensate. Revista Internacional de Medicina y Ciencias de la Actividad Física y el Deporte vol. 20 (77) pp. 197-210 Http://cdeporte.rediris.es/revista/revista78/artnitritos1141.htm DOI: http://doi.org/10.15366/rimcafd2020.78.001

\title{
ORIGINAL
}

\section{NADAR 2,5-KM INDOOR DISMINUYE EL NITRITO Y pH EN AIRE ESPIRADO CONDENSADO}

\section{SWIM 2,5-KM INDOOR DECREASES THE NITRITE AND PH IN EXHALED BREATH CONDENSATE}

\author{
Araneda, O.F.'; Calderón A. ${ }^{2}$; Terreros, L. ${ }^{3}$ y Cavada, G. ${ }^{4}$ \\ ${ }^{1}$ M.D., Ph.D. Laboratorio de Fisiología Integrativo de Biomecánica y Fisiología Integrativa (LIBFE), \\ Escuela de Kinesiología, Facultad de Medicina, Universidad de los Andes (Chile) \\ ofaraneda@miuandes.cl \\ ${ }^{2}$ M.Sc. Universidad de la Frontera, Campus Pucón (Chile) andrecalderon@gmail.com \\ 3 M.Sc. Dirección de Desarrollo Comunitario, Departamento de Deportes, Municipalidad de \\ Providencia. Departamento de Eventos, Deportes y Recreación, Municipalidad de Las Condes \\ (Chile) luzterreros@gmail.com \\ ${ }^{4}$ Ph.D. Escuela de Salud Pública, Facultad de Medicina Universidad de Chile. Facultad de Medicina \\ Universidad Finis Terrae (Chile) gcavada@med.uchile.cl
}

Código UNESCO / UNESCO Code: 2411.06 Fisiología del Ejercicio / Exercise Physiology

Clasificación Consejo de Europa / Council of Europe Classification: 2: Bioquímica del deporte / Biochemistry Sport, 6: Fisiología del ejercicio / Exercise Physiology

Recibido 5 de junio de 2018 Received June 5, 2018

Aceptado 15 de febrero de 2020 Accepted February 15, 2020

\section{RESUMEN}

OBJETIVO: determinar el efecto de una prueba aeróbica de natación en piscina clorada indoor sobre la concentración de $\mathrm{NO}_{2}^{-}, \mathrm{H}_{2} \mathrm{O}_{2}$ y el pH en el condensado del aire espirado. MÉTODO: diez nadadores aficionados nadaron 2,5 km en piscina clorada. Se obtuvieron muestras antes y en cuatro oportunidades durante las ocho horas posteriores a la prueba. El análisis estadístico usó modelos mixtos y la prueba de Spearman RESULTADOS: la prueba se realizó a 74,99 $\pm 10,10 \%$ de la reserva 
cardíaca y duró $50,80 \pm 8,98$ minutos. Posterior a la prueba disminuyó el $\mathrm{NO}_{2}{ }^{-}$ $(p=0,04)$ y el $\mathrm{pH}(p=0,02)$ en el condensado del aire espirado. Los valores preejercicio se relacionaron con los cambios absolutos $p=0,0002, p=0,047$ y con el volumen de entrenamiento $p=0,017, p=0,077$ para $\mathrm{NO}_{2}^{-}$y $\mathrm{H}_{2} \mathrm{O}_{2}$ respectivamente. CONCLUSIONES: la natación en piscina clorada disminuye la concentración de $\mathrm{NO}_{2}{ }^{-}$y el $\mathrm{pH}$ en el condensado del aire espirado.

PALABRAS CLAVE: natación, daño oxidativo, condensado del aire espirado

\section{ABSTRACT}

OBJECTIVE: to determine the effect of an aerobic swimming test in chlorinated indoor swimming pool on the concentration of $\mathrm{NO}_{2}^{-}, \mathrm{H}_{2} \mathrm{O}_{2}$ and the $\mathrm{pH}$ in the exhaled breath condensate. METHODS: ten amateur swimmers swam 2,5 km in a chlorinated pool. Samples were obtained before and four times in the eight hours after the test. The statistical analysis used mixed models and the Spearman test. RESULTS: the test was performed at $74,99 \pm 10,10 \%$ of the cardiac reserve and lasted $50,80 \pm 8,98$ minutes. After the test the $\mathrm{NO}_{2}^{-}(p=0,04)$ and the $\mathrm{pH}(\mathrm{p}=$ $0,02)$ in the exhaled air condensate decreased. The pre-exercise values were related to the absolute changes $p=0,0002, p=0.047$ and with the training volume $p$ $=0,017, p=0.077$ for $\mathrm{NO}_{2}$ and $\mathrm{H}_{2} \mathrm{O}_{2}$ respectively. CONCLUSIONS: Swimming in a chlorinated pool decreases the $\mathrm{NO}_{2}{ }^{-}$concentration and the $\mathrm{pH}$ in the exhaled breath condensate.

KEYWORDS: swimming, oxidative damage, exhaled breath condensate.

\section{INTRODUCCIÓN}

Durante el ejercicio el sistema respiratorio sufre grandes modificaciones de su funcionalidad. Así, aumenta tanto la llegada de sangre al compartimiento vascular como aire a la vía aérea. Este último sector, normalmente genera un ambiente protegido a las células epiteliales, en cuanto a temperatura y humedad, que puede verse afectado en el ejercicio, vía la evaporación acelerada, por la caída de la temperatura y eventualmente por la mayor llegada de irritantes como aire frio, polen, material particulado y gases ambientales. Esto finalmente genera una inflamación localizada, altera el estado redox tisular y puede afectar la función pulmonar (Araneda \& Tuesta, 2016). En los últimos años, nuestro grupo se ha dedicado al estudio de este fenómeno, usando el análisis de muestras del condensado del aire espirado (CAE). Así, hemos reportado incrementos del $\mathrm{H}_{2} \mathrm{O}_{2}$ y malondialdehído en escaladores (Araneda et al., 2005) y sujetos que entrenan a mediana altura (Heinicke et al., 2009). Además, hemos observado el incremento de pro-oxidantes en corredores urbanos en distancias sobre $21 \mathrm{~km}$ (Araneda et al., 2012). Posteriormente, reportamos aumentos localizados de concentración de nitritos y 
peróxido de hidrógeno en el CAE en personas activas que corrieron $10 \mathrm{~km}$ en pista abierta (Araneda et al., 2014). Finalmente, en condiciones de laboratorio, encontramos que a mayor tiempo de ejercicio aumenta la producción de estas mismas especies químicas (Tuesta et al., 2016).

Un escenario ambiental ampliamente usado para la realización de ejercicio corresponde al de las piscinas que utilizan cloro como desinfectante del agua, en vista de los reconocidos efectos que este ambiente genera en piel y mucosas de los nadadores, por esta razón resulta relevante estudiar los efectos pulmonares en sus usuarios (Davies et al., 2018; Cavaleiro et al., 2018) . Previamente, en sujetos que nadaron 40 minutos, se encontró ausencia de cambios en la concentración de 8isoprostanos y en citoquinas medidas en CAE, con un aumento de la proteína secretora de células claras (CC16) en suero que sugiere un incremento de la permeabilidad epitelial (Font-Ribera et al., 2010). En un reporte posterior, se describió un alza de la concentración de 8-isoprostanos en CAE, luego de 100 minutos de entrenamiento (Morissette et al., 2016). En vista de estos antecedentes, nos planteamos la pregunta: ¿Cuál es el efecto de la realización de ejercicio agudo en piscina clorada sobre la producción de pro-oxidantes y el pH en el CAE?, para responderla, realizamos una prueba de natación en piscina clorada techada, en condiciones ambientales habituales de concentración de humedad, temperatura y concentración de cloro en nadadores con un seguimiento de hasta ocho horas posterior a la prueba.

\section{MATERIAL Y MÉTODOS}

\section{PARTICIPANTES}

10 nadadores aficionados, cuyas características se resumen en la tabla uno. No fumadores, sin historia de rinitis o asma, sin infecciones respiratorias durante el último mes. Tampoco eran usuarios de antiinflamatorios, antioxidantes ni algún otro suplemento nutricional. Los participantes fueron informados oralmente y por escrito del protocolo, antes de dar su consentimiento por escrito. Este estudio se llevó a cabo resguardando los principios éticos que rigen la legislación nacional de Chile y según los principios de la declaración de Helsinki. 


\begin{tabular}{|c|c|}
\hline Parámetro & Valores \\
\hline Sexo (Hombres/Mujeres) & $7 / 3$ \\
\hline Edad (años) & $27,9 \pm 13,1$ \\
\hline Altura (m) & $1,64 \pm 0,51$ \\
\hline Peso (kg) & $62,6 \pm 9,2$ \\
\hline $\begin{array}{c}\text { Índice de masa corporal } \\
\text { (kg/m }{ }^{2} \text { ) }\end{array}$ & $22,3 \pm 2,8$ \\
\hline Experiencia (años) & $4,0 \pm 2,1$ \\
\hline $\begin{array}{c}\text { Volumen de entrenamiento } \\
\text { (horas/semana) }\end{array}$ & $12,6 \pm 10,7$ \\
\hline $\begin{array}{c}\text { Frecuencia de entrenamiento } \\
\text { (días/semana) }\end{array}$ & $5,7 \pm 0,9$ \\
\hline
\end{tabular}

Tabla 1. Características generales de los participantes. Los datos son presentados como promedio \pm desviación standard.

\section{PROCEDIMIENTOS}

Previo a la prueba, los participantes no realizaron actividad física intensa durante 48 horas. El día del test, se presentaron a las 7:00 AM habiendo desayunado una hora antes de la prueba. Allí se determinó la frecuencia cardíaca de reposo, estando acostados y previo 5 minutos de descanso. Luego de esto, las muestras de CAE fueron tomadas, en reposo, previo lavado bucal con agua destilada y usando un clip nasal. Para esto se utilizó el equipo condensador de aire espirado descrito previamente (Araneda et al., 2005; Araneda \& Salazar, 2009). El tiempo total de recolección fue aproximadamente 15 minutos, periodo en el que se obtuvo cerca de $1,5 \mathrm{ml}$ de muestra. Finalizado este procedimiento, la muestra fue almacenada en nitrógeno líquido y posteriormente transferida a $-80^{\circ} \mathrm{C}$ hasta su análisis. Las muestras de CAE y la medición de la frecuencia cardíaca fueron realizadas, previo al ejercicio (pre) y a los 20,180, 360 y 480 minutos luego de este. Posterior a la primera toma de muestra de CAE, los participantes, realizaron un calentamiento que consistió en nadar libremente durante 200 metros al $60 \%$ de la frecuencia cardíaca máxima calculada como 220 -edad. Una vez concluido este período realizaron una prueba maximal de 2500 metros de natación estilo "front crawl", deteniéndose cada 500 metros para determinar, durante un minuto la frecuencia cardíaca por un examinador. Las condiciones ambientales durante la prueba fueron una temperatura del agua de $27,0 \pm 1,7^{\circ} \mathrm{C}$ y una humedad ambiental relativa de $56,0 \pm 6,4 \%$. El cloro libre residual se mantuvo en rango de 0,5 a 1,5 ppm según la norma del centro acuático. 
Peróxido de hidrógeno: esta sustancia química se midió en el CAE usando el reactivo $\mathrm{FOX}_{2}$ (Nourooz-Zadeh et al., 1994). Este reactivo contiene $\mathrm{Fe}^{+2}(250 \mu \mathrm{M})$, que en un medio ácido $\left(\mathrm{HClO}_{4}, 110 \mathrm{mM}\right)$ se oxida a $\mathrm{Fe}^{+3}$ por la presencia de $\mathrm{H}_{2} \mathrm{O}_{2}$. La cantidad de $\mathrm{H}_{2} \mathrm{O}_{2}$ se monitoreó mediante la reacción entre el ion férrico y el indicador de anaranjado de xilenol $(250 \mu \mathrm{M})$, además, a la reacción original se añadió sorbitol (100 mM) de acuerdo con Gay \& Gebicki (Gay \& Gebicki, 2002); este método ha sido utilizado previamente por nuestro grupo de investigación (Araneda et al., 2012;Araneda et al., 2014). Para las mediciones, se tomaron 350 $\mu \mathrm{L}$ de CAE y $150 \mu \mathrm{L}$ de $\mathrm{FOX}_{2}$, luego se incubó la muestra durante una hora a temperatura ambiente y se leyó la absorbancia a $560 \mathrm{~nm}$ en un lector de placa (EPOCHTM, BioTek Instruments, EE. UU.). Se realizaron tres curvas de calibración para cada grupo de mediciones utilizando $\mathrm{H}_{2} \mathrm{O}_{2}$ (Merck) como estándar.

Nitritos $\left(\mathrm{NO}_{2}{ }^{-}\right)$: La concentración de nitrito se midió usando la prueba espectrofotométrica basada en la reacción de Griess (Green et al., 1982), así, se añadieron $300 \mu \mathrm{L}$ de reactivo de Griess $(0,1 \%$ de naftiletilendiamina-dihidrocloruro, $1 \%$ de sulfanilamida, $3 \%$ de $\mathrm{H}_{3} \mathrm{PO}_{4}$ a $300 \mu \mathrm{L}$ de $\mathrm{CAE}$. La mezcla se incubó durante diez minutos y se midió la absorbancia a $550 \mathrm{~nm}$ usando el lector de placa mencionado previamente. Se hicieron tres curvas para cada medición, con nitrito de sodio como estándar.

pH: El pH se midió usando el método de Paget-Brown (Paget-Brown et al., 2006). Así, $100 \mu \mathrm{L}$ de CAE fueron burbujeados con Argón durante ocho minutos a un flujo de $350 \mathrm{~mL} / \mathrm{min}$, para luego medirse el $\mathrm{pH}$ usando un microelectrodo de $3 \times 38$ $\mathrm{mm}$ (Cole y Palmer) conectado a un medidor de pH (Oakton® Acorn pH 6).

\section{ANÁLISIS ESTADÍSTICOS}

Para estudiar la tendencia en el tiempo de los parámetros medidos en el CAE, se diseñó el siguiente modelo mixto:

$$
y=\beta \times \text { minuto }+\alpha
$$

Donde " $y$ " es el valor calculado del parámetro a estudiar, " $\alpha$ " es el intercepto y " $\beta$ " la pendiente de la curva. Las correlaciones fueron determinadas usando el test de Spearman, previa aplicación del test de Shapiro-Wilk para determinar el tipo de distribución de la muestra. En las correlaciones se calcularon los cambios absolutos $(\Delta)$ de los parámetros medidos en el CAE, restando al valor obtenido antes de la prueba, al promedio de los cuatro valores obtenidos post prueba, además al dividir este parámetro por el valor pre-ejercicio multiplicado por cien se obtuvo el cambio porcentual $(\% \Delta)$. El nivel de significancia usado fue de $p<0,05$. Para el análisis estadístico se utilizó el programa, Stata 14 y GraphPad Prism, USA. 


\section{RESULTADOS}

La prueba fue realizada a $161,56 \pm 11,45$ latidos/min, lo que equivale a un $74,99 \pm 10,10 \%$ de la reserva cardíaca, que a su vez tuvo un valor de $123,50 \pm 9,9$ latidos/min. El tiempo total de la prueba fue de 50,80 $\pm 8,98$ minutos. Respecto a los parámetros medidos en CAE, se observa una alta dispersión, como se aprecia en la tabla 2.

Tabla 2. Concentración de peróxido de hidrógeno, nitrito y pH en el condensado del aire espirado en participantes de una prueba de natación de 2,5 km en piscina clorada indoor, medidos antes

(pre) y una vez finalizada la prueba (post) a los 20, 180, 360 y 480 minutos. Los valores se expresan como mediana y rango intercuartílico.

\begin{tabular}{|c|c|c|c|c|c|}
\hline Parámetro & pre & 20min-post & $\mathbf{1 8 0 - p o s t}$ & $\mathbf{3 6 0}$-post & 480-post \\
\hline $\begin{array}{c}{\left[\mathrm{H}_{2} \mathrm{O}_{2}\right]_{\mathrm{CAE}}} \\
\boldsymbol{\mu m o l} \cdot \mathbf{I - 1}\end{array}$ & $\begin{array}{c}0,68 \\
(0,20-1,08)\end{array}$ & $\begin{array}{c}0,63 \\
(0,27-1,72)\end{array}$ & $\begin{array}{c}0,48 \\
(0,22-0,83)\end{array}$ & $\begin{array}{c}0,55 \\
(0,32-1,09)\end{array}$ & $\begin{array}{c}0,42 \\
(0,24-0,78)\end{array}$ \\
\hline $\begin{array}{c}{\left[\mathrm{NO}_{2}\right]_{\mathrm{CAE}}} \\
\boldsymbol{\mu m o l} \cdot \mathbf{I - 1}\end{array}$ & $\begin{array}{c}2,00 \\
(0,73-3,59)\end{array}$ & $\begin{array}{c}2,22 \\
(1,84-2,65)\end{array}$ & $\begin{array}{c}1,50 \\
(1,05-2,27)\end{array}$ & $\begin{array}{c}1,91 \\
(1,45-2,90)\end{array}$ & $\begin{array}{c}1,42 \\
(0,78-1,81)\end{array}$ \\
\hline $\mathbf{p H}$ & $\begin{array}{c}7,74 \\
(7,43-8,00)\end{array}$ & $\begin{array}{c}7,68 \\
(7,42-8,03)\end{array}$ & $\begin{array}{c}7,68 \\
(7,47-7,87)\end{array}$ & $\begin{array}{c}7,52 \\
(7,28-7,81)\end{array}$ & $\begin{array}{c}7,57 \\
(7,38-7,88)\end{array}$ \\
\hline
\end{tabular}

Según el modelo de tendencia obtenido usando la prueba de modelos mixtos, la $\left[\mathrm{NO}_{2}{ }^{-}\right]_{\text {CAE }}$ disminuye en el período de tiempo analizado; $\alpha=-0,0019$ y $\beta=2,86$, con un $\mathrm{p}=0,044$ (ver figura 1b). Igual comportamiento fue encontrado para el $\mathrm{pH}_{\mathrm{CAE}}$; $\alpha=-0,00055$ y $\beta=7,69$ con un $p=0,021$ (ver figura 1a). Para la $\left[\mathrm{H}_{2} \mathrm{O}_{2}\right]_{\mathrm{CAE}}$, el modelo tuvo un valor de $\alpha=-0,0005$ y $\beta=0,98$ sin alcanzar la significancia estadística $(p=0,17)$. Respecto a las correlaciones, se encontraron asociaciones entre la duración de la prueba versus la frecuencia cardíaca promedio $(r=-0,74, p=0,016)$, volumen de entrenamiento medido en horas a la semana $(r=-0,77, p=0,010)$ como se observa en la figura $2 a$ y el porcentaje de la reserva cardíaca utilizada durante la prueba $(r=-0,65, p=0,049)$. La edad de los participantes mostró una tendencia a la significancia con el volumen de entrenamiento $(r=-0,62, p=0,06)$ como se observa en el figura 2b. Respecto a los metabolitos medidos en el CAE, los valores de la $\left[\mathrm{NO}_{2}{ }^{-}\right]_{\mathrm{CAE}}$ pre ejercicio correlacionaron con el volumen de entrenamiento $(\mathrm{r}=-0,74$, $\mathrm{p}=0,017)$ como se aprecia en la figura $2 \mathrm{c}$, con $\Delta\left[\mathrm{NO}_{2}{ }^{-}\right] \mathrm{CAE}(\mathrm{r}=-0,94, \mathrm{p}=0,0002)$, como se observa en la figura $3 b$ y con el $\% \Delta\left[\mathrm{NO}_{2}{ }^{-}\right]_{C A E}(r=-0,88, p=0,002)$. Los valores de $\Delta\left[\mathrm{NO}_{2}{ }^{-}\right]_{\text {CAE }}$ correlacionaron con el volumen de entrenamiento $(r=0,78, p=0,01)$. Respecto al \% $\Delta\left[\mathrm{NO}_{2}{ }^{-}\right]_{\mathrm{CAE}}$ correlacionó con el volumen de entrenamiento $(\mathrm{r}=0,76$, $\mathrm{p}=0,015)$ y la edad $(\mathrm{r}=-0,66, \mathrm{p}=0,044)$. La $\left[\mathrm{H}_{2} \mathrm{O}_{2}\right] \mathrm{CAE}$ pre ejercicio correlacionó con el $\Delta\left[\mathrm{H}_{2} \mathrm{O}_{2}\right]_{\mathrm{CAE}}(r=-0,83, p=0,005)$, como se ve en el figura 3 a y el $\% \Delta\left[\mathrm{H}_{2} \mathrm{O}_{2}\right]_{\mathrm{CAE}}$ $(r=-0,76, p=0,015)$, además mostró una tendencia a la significancia con el volumen 
de entrenamiento $(r=-0,58, p=0,077)$ como se muestra en la figura $2 d$. Los valores de $\Delta\left[\mathrm{H}_{2} \mathrm{O}_{2}\right]_{\text {CAE }}$ correlacionaron con $\Delta\left[\mathrm{NO}_{2}{ }^{-}\right]_{\text {CAE }}(\mathrm{r}=0,86, \mathrm{p}=0,003)$, mientras que el $\% \Delta\left[\mathrm{H}_{2} \mathrm{O}_{2}\right]_{\text {CAE }}$ lo hizo con $\% \Delta\left[\mathrm{NO}_{2}^{-}\right]_{\text {CAE }}(\mathrm{r}=0,79, \mathrm{p}=0,009)$. El valor de $\mathrm{pH}$ CAE pre ejercicio correlacionó con el porcentaje de frecuencia cardíaca máxima promedio durante la prueba $(r=-0,75, p=0,015)$, mientras tendió a la significancia con el porcentaje de la reserva cardíaca $(r=-0,62, p=0,060)$. Los valores de $\Delta \mathrm{pH}_{\text {CAE }}(\mathrm{r}=0,73$ $p=0,021)$ y los valores de $\% \Delta \mathrm{pH}_{\mathrm{CAE}}(\mathrm{r}=0,69, \mathrm{p}=0,035)$ durante la prueba se asociaron con la edad. Finalmente, se correlacionaron todos los valores $(n=50)$ de los parámetros medidos en CAE, así se encontró una correlación directa entre $\left[\mathrm{NO}_{2}{ }^{-}\right.$ ]$_{\text {CAE versus }}\left[\mathrm{H}_{2} \mathrm{O}_{2}\right]_{\text {CAE }}(\mathrm{r}=0,78, \mathrm{p}<0,0001)$.

a

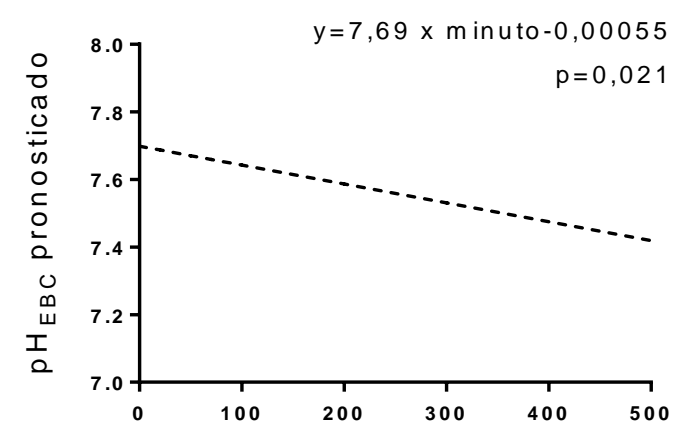

b

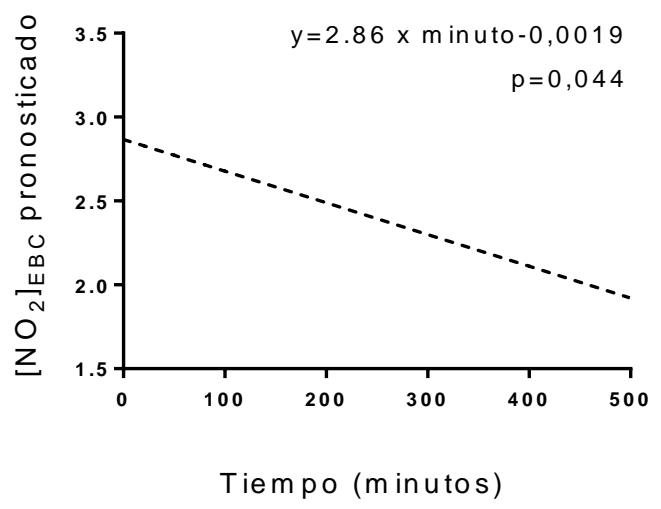

Figura 1. Modelos mixtos propuestos para la variación de $\mathrm{pH}_{\mathrm{CAE}}(\mathrm{a})$ y $\left[\mathrm{NO}_{2}\right]_{\mathrm{CAE}}$ (b), construidos a partir de los valores pre y los valores obtenidos en las cuatro etapas de medición post ejercicio durante ocho horas. 
a

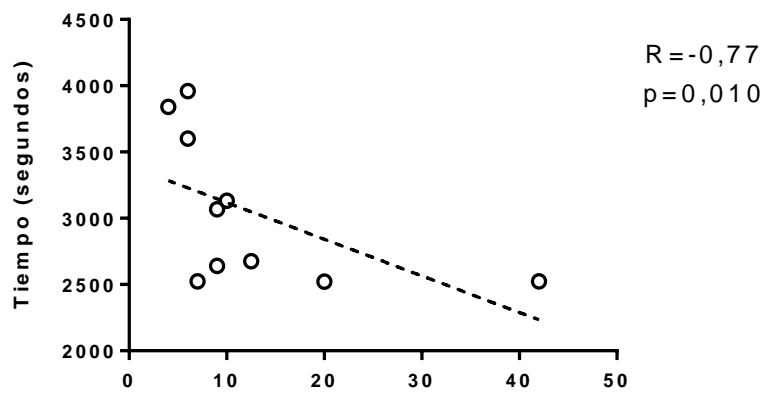

b

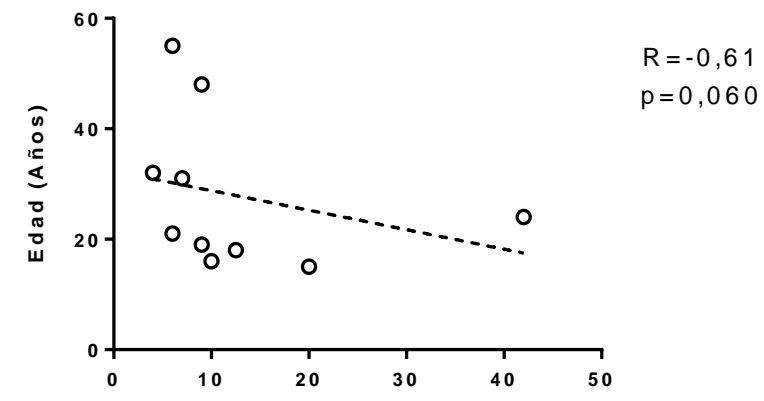

C
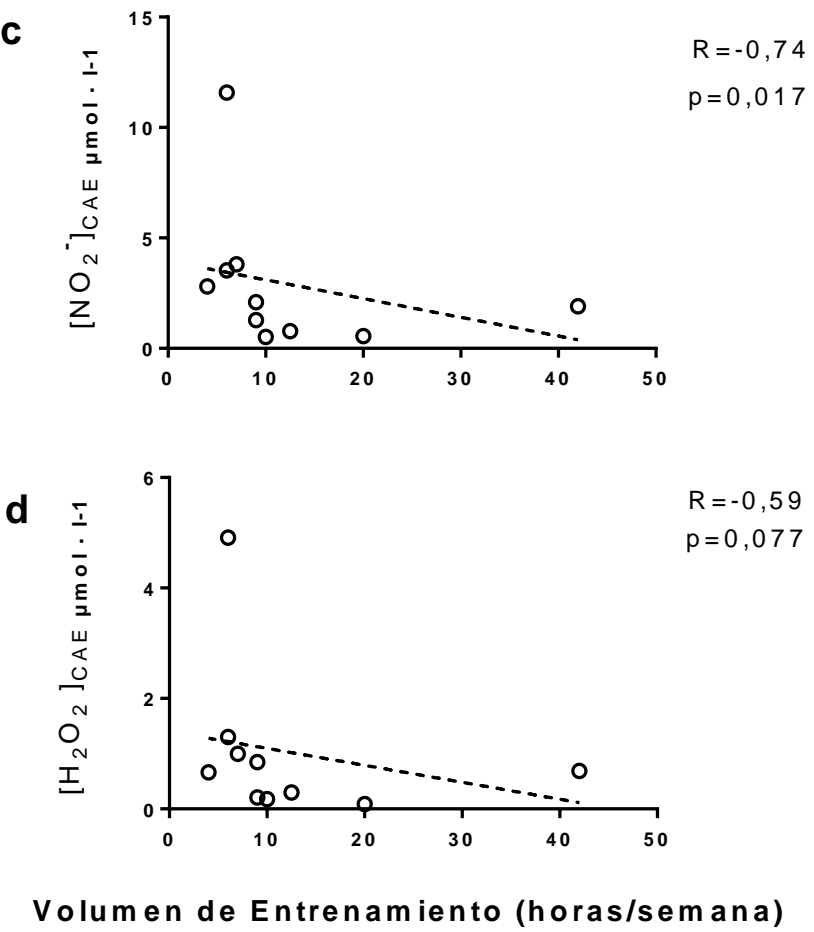

Figura 2. Relación entre el volumen de entrenamiento físico en piscina versus tiempo durante la prueba de 2,5 km de natación (a), edad (b), $\left[\mathrm{NO}_{2}\right]$ CAE pre (c) y $\left[\mathrm{H}_{2} \mathrm{O}_{2}\right]$ CAE pre(d). 
a

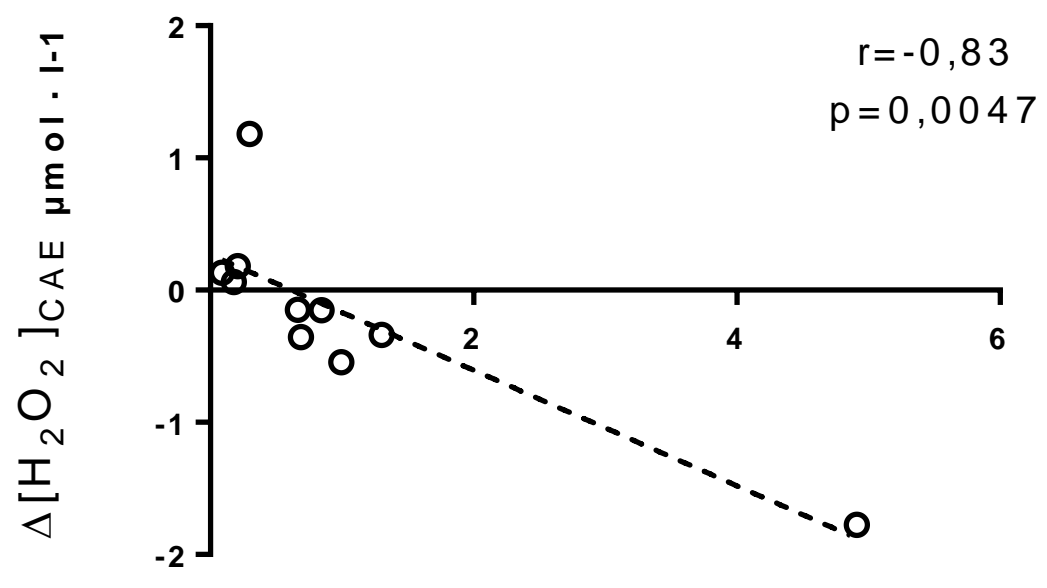

$\left[\mathrm{H}_{2} \mathrm{O}_{2}\right]_{E B C \mu \mathrm{mol} \cdot \mathrm{I}-1}$

b

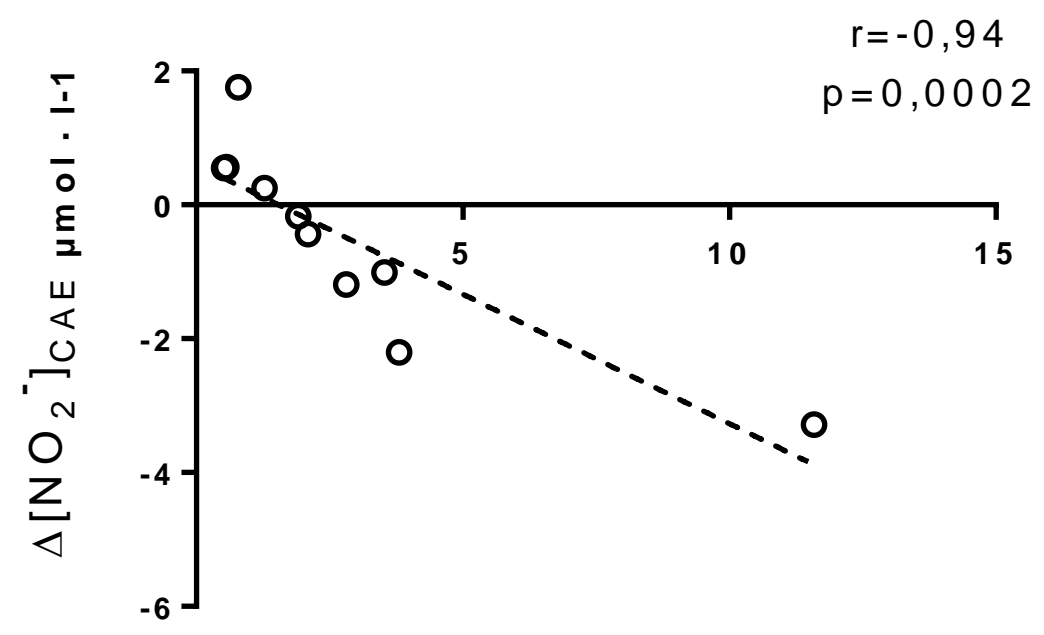

$\left[\mathrm{NO}_{2}^{-}\right]_{E B C \mu m o l} \cdot \mathrm{l}-1$

Figura 3. Relación entre los valores pre-ejercicio versus los cambios absolutos de $\left[\mathrm{H}_{2} \mathrm{O}_{2}\right]$ CAE (a) y de $\left[\mathrm{NO}_{2}\right]_{\text {CAE }}$ (b) generados por la prueba de $2,5 \mathrm{~km}$ de natación en piscina techada. Los valores post ejercicio corresponden al promedio de las cuatro muestras tomadas luego de la prueba.

\section{DISCUSIÓN}

En el presente reporte, nadadores realizaron una prueba de $2,5 \mathrm{~km}$ en piscina clorada techada. Luego de la prueba, se encontró una baja en la $\left[\mathrm{NO}_{2}^{-}\right]_{\mathrm{CAE}}$, baja del $\mathrm{pH}_{\mathrm{CAE}}$, sin cambios en la $\left[\mathrm{H}_{2} \mathrm{O}_{2}\right]_{\text {CAE }}$ durante las ocho horas posteriores al estímulo. 
En comparación a nuestros resultados anteriores, esta tendencia general es novedosa en vista de la disminución del nitrito, un conocido pro-oxidante de la vía aérea y al favorecimiento de un potencial cuadro inflamatorio localizado medido como el pH $\mathrm{H}_{\mathrm{CAE}}$. En vista de lo expuesto, nuestros resultados son en parte contrarios a la hipótesis inicial, ya que esperábamos que la combinación de ejercicio intenso; cerca del $75 \%$ de la reserva cardíaca por casi una hora, y el contacto con el cloro y sus derivados, tanto vaporizados como disueltos en el agua de la piscina, serían fuente de un incremento de los pro-oxidantes y promotores de la inflamación.

Los pro-oxidantes evaluados en el CAE $\left(\mathrm{H}_{2} \mathrm{O}_{2}\right.$ y NO$\left.{ }_{2}^{-}\right)$, son especies químicas participantes tanto en funciones fisiológicas como la reactividad vascular, procesos de señalización intracelular (Magherini et al., 2019) como patológicas al ser mediadores del proceso inflamatorio, que han sido encontradas en mayor concentración en CAE en el asma, la fibrosis quística, la enfermedad pulmonar obstructiva crónica o luego de fumar (Konstantinidi et al., 2015; Causer et al., 2020). En el ejercicio, los protocolos de corta duración no han reportado cambios en la $\left[\mathrm{H}_{2} \mathrm{O}_{2}\right]$ CAE (Nowak et al., 2001, Marek et al., 2008). Cuando el ejercicio se realiza por un tiempo superior a dos horas en carreras pedestres al aire libre (Araneda et al., 2012) o sobre noventa minutos en cicloergómetro en condiciones de laboratorio (Araneda et al., 2016) hemos encontrado habitualmente incrementos tanto de la $\left[\mathrm{H}_{2} \mathrm{O}_{2}\right]_{\text {CAE }}$ como de la $\left[\mathrm{NO}_{2}{ }^{-}\right]_{\text {CAE }}$ a los 20 y 80 minutos posteriores al cese del ejercicio. Como ya fue mencionado, estos hallazgos son contrarios a los presentados en este trabajo, donde el modelo obtenido evidenció una baja de la $\left[\mathrm{NO}_{2}{ }^{-}\right]_{\text {CAE }}$ durante las ocho horas posteriores al ejercicio. En uno de los escasos modelos experimentales similares al aquí presentado, Font Ribera, reportó una tendencia a la disminución del óxido nítrico (del que derivan los nitritos), medido directo en el aire exhalado, luego de nadar 40 minutos en piscina clorada por parte de un grupo de sujetos atópicos (Font Ribera et al., 2010). Respecto a la $\left[\mathrm{H}_{2} \mathrm{O}_{2}\right]_{\mathrm{CAE}}$, encontramos una gran variabilidad, razón por la que probablemente no se alcanzó la significancia, sin embargo, es probable que esta especie química, se comporte de manera similar a la $\left[\mathrm{NO}_{2}^{-}\right]$CAE por las correlaciones estrechas que hemos encontrado entre ellos (ver resultados). Una explicación a nuestros resultados nos obliga a buscar las diferencias con otros modelos de ejercicio y condiciones ambientales previamente publicadas. Así al nadar, se desarrolla una respiración en ciclos voluntarios, lo que implica una ventilación total pulmonar de menor cuantía respecto a las carreras pedestres. Esto implicaría una menor caída de la temperatura de la vía aérea y una menor deshidratación epitelial. Además, la prueba fue desarrollada en condiciones de mayor temperatura y humedad propia de las piscinas techadas. Así, esto genera la disminución de la diferencia epitelio/ambiente, provocando menores pérdidas de agua y con ello aminorando el daño celular. A este teórico escenario favorable respecto a que se estimula menos la formación de pro-oxidantes vía irritación, se suma el incremento de los sistemas antioxidantes (enzimáticos/no enzimáticos) en su actividad/concentración luego de 
la realización de ejercicio (Powers et al., 2014), que en parte, pueden aportar a explicar lo encontrado.

En otro aspecto, la inflamación pulmonar ha sido estudiada mediante la determinación del $\mathrm{pH}_{\mathrm{CAE}}$. Así, se ha descrito la disminución de su valor en el caso de procesos inflamatorios pulmonares (Hunt, 2006). En nuestro actual reporte, hemos encontrado menores valores de $\mathrm{pH}_{\mathrm{CAE}}$ hasta ocho horas posteriores al ejercicio. Este hallazgo, se encuentra en la dirección de lo que esperábamos, y corresponde a un hallazgo interesante, en vista que previamente, se había descrito una tendencia a la alcalosis post ejercicio, como lo reportó Riediker y Danuser en un ejercicio en treadmill al $60 \%$ de la frecuencia cardíaca máxima (Riediker \& Danuser, 2007). En la misma dirección, también encontramos una tendencia al incremento de $\mathrm{pH}_{\mathrm{CAE}}$ en participantes en dos pruebas de $10 \mathrm{~km}$ realizadas a máxima intensidad, tanto en no entrenados (Araneda et al., 2014) como en corredores habituales (Araneda et al., 2012). Esta tendencia es de sumo interés, en vista que el tiempo en la distancia mencionada, es similar al ocupado en la prueba de natación reportada actualmente. En este sentido, nuestro actual hallazgo es más homologable a la tendencia a la baja del pH CAE que hemos visto en carreras pedestres de más de dos horas de duración, en las distancias de 21 y $42 \mathrm{~km}$ respectivamente. Lo anterior, nos hace pensar que probablemente se agregó un nuevo factor al ejercicio como promotor de la baja del pH CAE. Así, es posible que este se trate del contacto directo con los derivados vaporizados del cloro presentes en la piscina, ya que estas especies actúan como irritantes de la superficie epitelial. A pesar de que esta hipótesis es plausible, nuestro modelo experimental es insuficiente para comprobarla y deberá ser evaluada posteriormente.

En otro aspecto, en nuestra muestra es posible observar una importante dispersión de la edad (ver tabla 1), además se observa que los sujetos más entrenados, mostraron un mejor rendimiento en la prueba, mostrando una tendencia a ser menores en edad como se observa en la figura $2 \mathrm{a}$ y $2 \mathrm{~b}$ respectivamente. En los marcadores químicos medidos en el CAE, se aprecia que los valores basales de ambos pro-oxidantes están estrechamente relacionados, de manera inversa, con los cambios absolutos generados por el ejercicio, como se observa en la figura $3 a$ y $3 \mathrm{~b}$, por esta razón era relevante caracterizar los factores con los cuales estos pudieran estar relacionados. Así, en nuestra muestra, los nadadores con menor cantidad de horas de entrenamiento a la semana poseían mayores concentraciones de la $\left[\mathrm{NO}_{2}{ }^{-}\right]_{\mathrm{CAE}}$ basal (ver figura $2 \mathrm{~d}$ ) y probablemente eran mayores en edad. En el caso de la $\left[\mathrm{H}_{2} \mathrm{O}_{2}\right]_{C A E}$ (ver figura $2 \mathrm{c}$ ), donde no se alcanza la significancia $(p=0,077)$, la tendencia es similar al anterior. Esta idea se fortalece aún más por la estrecha correlación entre los valores de $\left[\mathrm{NO}_{2}{ }^{-}\right]_{\text {CAE versus }}\left[\mathrm{H}_{2} \mathrm{O}_{2}\right]_{\mathrm{CAE}}$ como fue descrito en los resultados. En todo caso, no es posible descartar que la edad tenga también alguna influencia sobre los valores basales de los pro-oxidantes, sin embargo, el bajo número de participantes nos impide aislar este efecto y deberá ser estudiado a futuro. En el caso del valor basal de pH $\mathrm{CH}_{\mathrm{CE}}$, tenemos sólo evidencia indirecta que 
pudiera estar relacionado con el volumen de entrenamiento, ya que correlaciona inversamente con dos parámetros medidos en la prueba; el porcentaje de la reserva cardíaca utilizada y la frecuencia cardíaca promedio que se asocian significativamente con el tiempo empleado en la prueba, de ser esto así, implicaría que los sujetos más entrenados tendrían un menor valor basal de $\mathrm{pH}_{\mathrm{CAE}}$. Este hallazgo es similar a lo descrito en corredores de fondo, donde se descrito que los sujetos con más horas de entrenamiento poseen un menor pH $\mathrm{H}_{\mathrm{CAE}}$ basal (Ferdinands et al., 2008).

Respecto a los cambios post ejercicio, en los pro-oxidantes los sujetos que tienen mayores valores basales (que entrenan menos) presentan las mayores caídas, tanto de la $\left[\mathrm{H}_{2} \mathrm{O}_{2}\right]_{\text {CAE }}$ (ver figura $3 \mathrm{a}$ ) como de la $\left[\mathrm{NO}_{2}{ }^{-}\right]_{\mathrm{CAE}}$ (ver figura $3 \mathrm{~b}$ ). Este resultado puede relacionarse con hallazgos previos, donde las personas que poseían un mayor nivel de entrenamiento presentan menores cambios en carreras de $10 \mathrm{~km}$, respecto a los no entrenados (Araneda et al., 2104), aunque en el actual reporte, la tendencia de los valores post prueba es a la baja. Existen, además, dos correlaciones respecto a la edad que pueden aportar a reafirmar que participantes más jóvenes, que estaban más entrenados y que trabajaron a una mayor intensidad durante la prueba, presentaron menores cambios tanto en el $\% \Delta \mathrm{pH}_{\mathrm{CAE}}$ y en el $\% \Delta$ $\left[\mathrm{NO}_{2}{ }^{-}\right]_{\text {CAE }}$ respectivamente. Otro aspecto novedoso respecto a nuestros resultados anteriores corresponde a la pérdida de la relación entre la producción de prooxidantes y los cambios en el $\mathrm{pH}_{\mathrm{CAE}}$ con una tendencia a la baja observadas en ejercicio (Araneda et al., 2014; Araneda et al., 2012), entonces ante la ausencia de este efecto, incluso con pro-oxidantes a la baja como hemos descrito, es posible que otros factores expliquen la baja del pH $\mathrm{pAE}$, como el contacto directo con derivados del cloro que fue comentado más arriba. Finalmente, son limitaciones de este estudio el bajo número de participantes, en vista de la conocida alta variabilidad de las determinaciones en el CAE. En segundo término, faltan determinaciones espirométricas que podrían aportar a dilucidar eventuales cambios funcionales pulmonares.

\section{CONCLUSIÓN}

En un grupo de nadadores sanos, luego de una prueba de 2,5 $\mathrm{km}$ en piscina clorada indoor disminuyen la concentración de nitrito y el pH en el condensado del aire espirado hasta ocho horas posteriores a la natación, en este fenómeno, en parte, pueden estar implicados la mayor temperatura y humedad propias de las piscinas indoor. Los resultados aquí obtenidos también hacen vislumbrar la potencial utilidad de estos marcadores para realizar monitoreo en nadadores de piscina clorada.

\section{REFERENCIAS BILBIOGRÁFICAS}

Araneda, OF., García, C., Lagos, N., Quiroga, G., Cajigal, J., Salazar, M.P., \& Behn, C. (2005). Lung oxidative stress as related to exercise and altitude. Lipid 
peroxidation evidence in exhaled breath condensate: a possible predictor of acute mountain sickness. Eur J Appl Physiol,95, 383-90.

Araneda O.F., Carbonell T., Tuesta M. (2016). Update on the Mechanisms of Pulmonary Inflammation and Oxidative Imbalance Induced by Exercise. Oxid Med Cell Longev, 4868536.

Araneda, O.F. \& Salazar, M.P. (2009). Design and evaluation of a device for collecting exhaled breath condensate. J Bras Pneumol, 35, 69-72.

Araneda O.F., Urbina-Stagno R., Tuesta M., Haichelis D., Alvear M., Salazar M.P., García C. (2014). Increase of pro-oxidants with no evidence of lipid peroxidation in exhaled breath condensate after a $10-\mathrm{km}$ race in nonathletes. J Physiol Biochem, 70(1), 107-15.

Causer, A. J., Shute, J. K., Cummings, M. H., Shepherd, A. I., Gruet, M., Costello, J. T., Bailey, S., Lindley, M., Pearson, C., Connett, G., Allenby, M. I., Carroll, M. P., Daniels, T., \& Saynor, Z. L. (2020). Circulating biomarkers of antioxidant status and oxidative stress in people with cystic fibrosis: A systematic review and meta-analysis. Redox biology, 101436. https://doi.org/10.1016/j.redox.2020.101436

Cavaleiro, Rufo J., Paciência, I., Silva, D., Martins, C., Madureira, J., Oliveira Fernandes, E., Padrão, P., Moreira, P., Delgado, L., Moreira, A. (2018) Swimming pool exposure is associated with autonomic changes and increased airway reactivity to a beta-2 agonist in school aged children: A cross-sectional survey. PLoS One,13(3), e0193848.

Davies, R. Parent, E., Steinback, C., Kennedy, M. (2018) The Effect of Different Training Loads on the Lung Health of Competitive Youth Swimmers. Int J Exerc Sci, 11(6),999-101.

Ferdinands, J.M., Crawford, C.G., Greenwald, R., David, Van Sickle, D., Hunter, E., \& Teague, W.G. (2008). Breath acidification in adolescent runners exposed to atmospheric pollution: A prospective, repeated measures observational study. Environmental Health, 7(10), 2008.

Font-Ribera, L., Kogevinas, M., Zock, J.P., Gómez, F.P., Barreiro, E., Nieuwenhuijsen, M.J., Fernandez, P., Lourencetti, C., Pérez-Olabarría, M., Bustamante, M., Marcos, R., Grimalt, J.O., \& Villanueva, C.M. (2010). Short-Term Changes in Respiratory Biomarkers after Swimming in a Chlorinated Pool. Environ Health Perspect, 118, 1538-1544.

Gay C., \& Gebicki J. (2002). Perchloric acid enhances sensitivity and reproducibility of the ferric-xylenol orange peroxide assay. Anal Biochem, 304(1), 42-6.

Green, L.C., Wagner, D.A., Glogowski, J., Skipper, P.L., Wishnok, J.S., \& Tannenbaum, S.R. (1982). Analysis of nitrate, nitrite, and [15N] nitrate in biological fluids. Anal Biochem, 126, 131-8.

Heinicke, I., Boehler, A., Rechsteiner, T., Bogdanova, A., Jelkmann, W., Hofer, M., Rawlings, P., Araneda, O.F., Behn, C., Gassmann, M., \& Heinicke, K. (2009). Moderate altitude but not additional endurance training increases markers of oxidative stress in exhaled breath condensate. Eur $J$ Appl Physiol, 106, 599-604. 
Hunt, J. (2007). Exhaled breath condensate pH assays. Immunol Allergy Clin North Am, 27, 597-606.

Konstantinidi E.M., Lappas A.S., Tzortzi A.S., Behrakis P.K. (2015) Exhaled Breath Condensate: Technical and Diagnostic Aspects. Scientific World Journal, 435160.

Magherini, F., Fiaschi, T., Marzocchini, R., Mannelli, M., Gamberi, T., Modesti, P. A., \& Modesti, A. (2019). Oxidative stress in exercise training: the involvement of inflammation and peripheral signals. Free radical research, 53(11-12), 1155-1165. https://doi.org/10.1080/10715762.2019.1697438

Marek, E., Mückenhoff, K., Streckert, H.J., Becher, G., \& Marek, W. (2008) Measurements of L-lactate and $\mathrm{H} 2 \mathrm{O} 2$ in exhaled breath condensate at rest and mild to moderate exercise in young and healthy subjects. Pneumologie, 62, 541-7.

Morissette M. , Murray N. , Turmel J., Milot J., Boulet L.P., Bougault V. (2016) Increased exhaled breath condensate 8-isoprostane after a swimming session in competitive swimmers. Eur J Sport Sci, 16(5), 569-76.

Nourooz-Zadeh, J., Tajaddini-Sarmadi, J., \& Wolf, S.P. (1994). Measurement of plasma hydroperoxide concentrations by the ferrous oxidation-xylenol orange assay in conjunction with triphenylphosphine. Anal Biochem, 220, 403-409.

Nowak, D., Kalucka, S., Białasiewicz, P., \& Król, M. Exhalation of $\mathrm{H}_{2} \mathrm{O}_{2}$ and thiobarbituric acid reactive substances (TBARs) by healthy subjects. (2001). Free Radic Biol Med, 15, 178-86.

Paget-Brown, A.O., Ngamtrakulpanit, L., Smith, A., Bunyan, D., Hom, S., Nguyen, A., \& Hunt, J.F. Normative data for $\mathrm{pH}$ of exhaled breath condensate. (2006). Chest, 129, 426-30.

Powers S.K., Sollanek K.J., Wiggs M.P., Demirel H.A., Smuder A.J. (2014). Exercise-induced improvements in myocardial antioxidant capacity: the antioxidant players and cardioprotection. Free Radic Res, 48(1), 43-51.

Riediker, M. \& Danuser, B. (2007). Exhaled breath condensate pH is increased after moderate exercise. $J$ Aerosol Med, 20, 13-8.

Tuesta, M., Alvear, M., Carbonell, T., García, C., Guzmán-Venegas, R., Araneda, O.F. (2016) Effect of exercise duration on pro-oxidants and $\mathrm{pH}$ in exhaled breath condensate in humans. J Physiol Biochem,72(2), 353-60.

Número de citas totales / Total references: 23 (100\%)

Número de citas propias de la revista / Journal's own references: $0(0 \%)$

Rev.int.med.cienc.act.fís.deporte - vol. 20 - número 78 - ISSN: 1577-0354 